\title{
Concurrent colon adenocarcinoma and hepatoid lung adenocarcinoma with normal serum level and negative immunostain of alpha-fetoprotein
}

\author{
Tyler Gutschenritter ${ }^{1}$, Lichao Zhao ${ }^{2}$, Chance Matthiesen ${ }^{3}$, Wajeeha Razaq and Mohammad Razaq* \\ ${ }^{1}$ College of Medicine, University of Oklahoma Health Sciences Center, Oklahoma City, USA \\ ${ }^{2}$ Department of Pathology, University of Oklahoma Health Sciences Center, Oklahoma City, USA \\ ${ }^{3}$ Department of Radiation Oncology, Stephenson Cancer Center, University of Oklahoma Health Sciences Center, Oklahoma City, USA \\ ${ }^{4}$ Hematology/Oncology Section, Department of Medicine, Stephenson Cancer Center, University of Oklahoma Health Sciences Center, Oklahoma City, USA
}

\begin{abstract}
Introduction: Hepatoid adenocarcinoma of the lung (HAL) is a rare clinical entity that accounts for only 5\% of hepatoid adenocarcinomas. Twenty-four cases of HAL have been documented in the medical literature, and the majority of cases share the following features: male predilection, history of smoking, large primary lung mass, advanced disease at presentation, poor prognosis, and elevated alpha-fetoprotein (AFP) serum levels.

Case presentation: We present a middle-aged male with locally advanced HAL who had normal serum AFP levels at the time of diagnosis, and whose recurrent tumor was not immunoreactive for AFP. Molecular testing for anaplastic lymphoma kinase (ALK) and epidermal growth factor receptor (EGFR) was unremarkable.

Discussion: To our knowledge, this is the third case of HAL to have normal serum levels of AFP and to not have positive AFP immunostaining. Moreover, this is the second case of HAL with a concurrent colon adenocarcinoma, but the first such case to have serum and immunohistochemical findings unremarkable for AFP. It is important to raise awareness of HAL in order to aid clinicians in distinguishing it from metastatic hepatocellular carcinoma (HCC). Given the poor prognosis of HAL, molecular testing should be utilized by clinicians to explore targeted treatment options. Specifically, ALK rearrangement and crizotinib therapy has been documented in an AFP-negative HAL. Lastly, clinicians should be familiar with the histological characteristics that discriminate HAL from HCC and know that the diagnosis of HAL does not require elevated AFP serum levels or immunohistochemical evidence of AFP.
\end{abstract}

\section{Introduction}

Hepatoid adenocarcinoma is an extrahepatic tumor with hepatocellular differentiation that most commonly occurs in the stomach [1]. Hepatoid adenocarcinoma of the lung (HAL) is a rare clinical entity that accounts for only $5 \%$ of hepatoid adenocarcinomas [1]. Twenty four cases of HAL have been documented in the medical literature, and the majority of cases share the following features: male predilection, history of smoking, large primary lung mass, advanced disease at presentation, poor prognosis, and elevated serum levels of AFP [2-5].

HAL was first defined as a clinical entity by Ishikura et al. in 1990 [6]. To meet Ishikura et al.'s diagnostic criteria for HAL, tumors must produce alpha-fetoprotein (AFP) and contain acinar or papillary adenocarcinoma as well as components with morphological resemblance to hepatocellular carcinoma, such as trabecular proliferation, eosinophilic cytoplasm, and hyaline globules [6]. Recently, Haninger et al. suggested modifying the diagnostic criteria for HAL by expanding the histological tumor composition needed for diagnosis and making AFP expression not requisite for diagnosis as long as other markers of hepatic differentiation are expressed [2].

Herein, we present a case of locally advanced, HAL in a middleaged white male with a 30 pack-year smoking history. Most notably, this patient had a coexisting colorectal adenocarcinoma along with a normal AFP serum level at the time of diagnosis and AFP-negative immunostaining of his recurrent HAL tumor. This is the first case of HAL to have a coinciding colorectal adenocarcinoma along with serum and immunohistochemical findings unremarkable for AFP.

\section{Case presentation}

In October 2013, a 57 year-old white male with a 30 pack-year smoking history was involved in a motorcycle crash on his way to our clinic for a staging colonoscopy of a sigmoid mass found during a routine colonoscopy. A CT following the crash revealed a $10.5 \mathrm{~cm}$ $\times 9.7 \mathrm{~cm} \times 10.4 \mathrm{~cm}$ left upper lobe lung mass with mediastinal and hilar lymphadenopathy. Biopsy revealed adenocarcinoma with predominantly large tumor cells containing relatively abundant eosinophilic cytoplasm arranged in insular and trabecular pattern with focal glandular formation. Immunohistochemistry stains were positive for hepatocyte paraffin 1 (HepPar1), heat shock protein-70 (HSP-70), glypican-3, cytokeratin 7 (CK7), but negative for CK20, CDX2, TTF-

Correspondence to: Mohammad Razaq Stephenson Cancer Center 800 NE 10th St. Oklahoma City, OK 73104 Phone: 405-271-4022 Fax: 405-271-3020; E-mail: mohammad-razaq@ouhsc.edu

Key words: hepatoid adenocarcinoma, lung cancer, colon cancer, concurrent cancer, alpha-fetoprotein (AFP)

Received: January 30, 2016; Accepted: February 22, 2016; Published: February 25,2016 
1 and Napsin A. These results suggested hepatocellular differentiation and ruled out colon cancer metastases. Biopsy of the sigmoid mass revealed a low grade, invasive adenocarcinoma negative for MLH1, MSH2, PMS2 and MSH6 by immunohistochemistry. Additionally, the lung tumor was negative for epidermal growth factor receptor (EGFR) mutation and anaplastic lymphoma kinase (ALK) translocation.

A PET scan demonstrated the previously described tumors but found no evidence of malignancy in the liver. Serum alpha-fetoprotein (AFP) was $2.6 \mathrm{ng} / \mathrm{mL}(.5-9 \mathrm{ng} / \mathrm{mL})$, and serum carcinoembryonic antigen (CEA) was $18.96 \mathrm{ng} / \mathrm{mL}(0-5 \mathrm{ng} / \mathrm{mL})$. The lung cancer was clinically staged as IIIB (cT4cN2). Elevated CEA was expected given the low grade colon adenocarcinoma, but the normal AFP serum level did not correlate with the hepatocellular differentiation of the lung tumor. A subsequent MRI of the liver was unremarkable which supported the diagnosis of hepatoid adenocarcinoma of the lung as opposed to metastatic hepatocellular carcinoma.

The patient was treated with chemoradiation involving 7 cycles of weekly carboplatin AUC 2 and paclitaxel $50 \mathrm{mg} / \mathrm{m} 2$ in conjunction with 70 Gy of external beam radiotherapy delivered via an intensity modulated radiation therapy (IMRT) technique. A chest CT scan prior to the final cycle of chemotherapy revealed the lung mass had decreased in size to $5.5 \mathrm{~cm} \times 6.1 \mathrm{~cm}$ while the involved lymph nodes had remained stable.

Following neoadjuvant therapy, the lung mass was resected with negative margins. All 17 sampled lymph nodes were negative for metastasis; therefore the tumor was pathologically staged as IIB (ypT3N0). Histology of the tumor revealed moderately differentiated adenocarcinoma with both morphologic and immunophenotypic evidence of hepatocellular differentiation and only minor component of acinar adenocarcinoma (Figure 1). No tissue staining for AFP was done at that time.

Following surgery, the patient had 2 cycles of adjuvant chemotherapy consisting of cisplatin and paclitaxel followed by 30 Gy of adjuvant radiation to the chest wall utilizing IMRT. Subsequent colonoscopy showed no residual malignancy, and a CT of the chest, abdomen, and pelvis was not concerning for malignancy. One year following completion of adjuvant treatment, the patient presented with chest wall pain, and a PET/CT revealed a $4.2 \mathrm{~cm} \times 5.6 \mathrm{~cm} \times$ $6.5 \mathrm{~cm}$ left anterior chest wall mass consistent with recurrence and multiple FDG-avid hilar and mediastinal lymph nodes. There was no

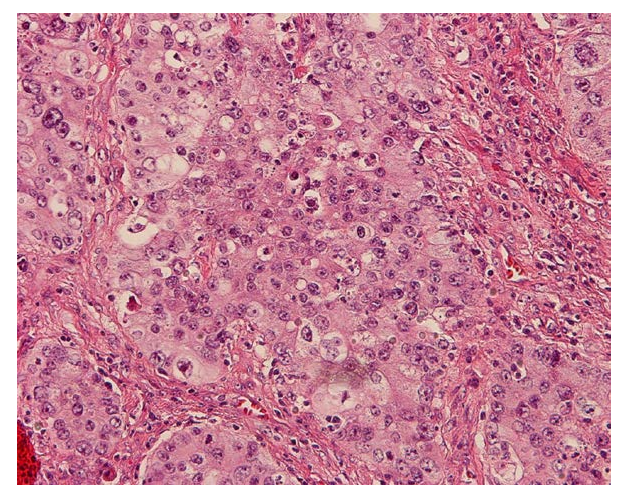

Figure 1. H\&E staining of resected tumor tissue at 200x magnification. Histology ofresected tumor shows predominantly large tumor cells with relatively abundan eosinophilic cytoplasm arranged in insular and trabecular pattern with focal glandular formation.

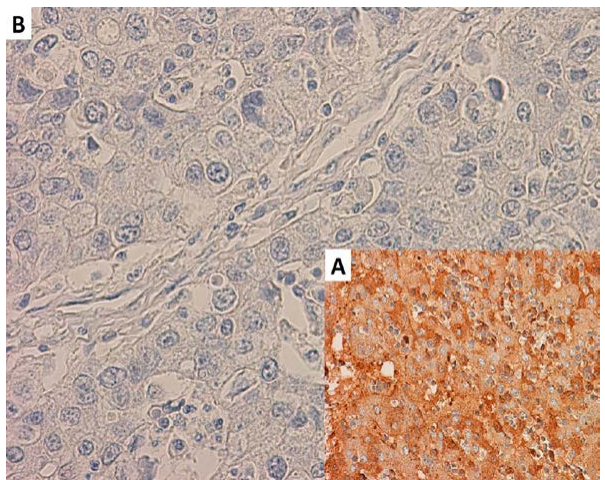

Figure 2. AFP staining of recurrent tumor compared to positive control at 400xmagnification. A) Positive control for AFP staining. B) Imunohistochemical staining forAFP was negative following resection of recurrent tumor.

evidence of distant metastatic disease. The chest wall mass was resected and found to be recurrent adenocarcinoma from the lung primary. Immunostaining confirmed the tumor was negative for AFP (Figure 2).

\section{Discussion}

Similar to the previously reported cases of HAL, our patient was a male with a smoking history who presented with a large tumor, had advanced disease, and whose tumor recurred following aggressive therapy. However, a unique feature of this case of HAL was its unremarkable AFP findings when examining the serum level and immunohistochemistry. To our knowledge, this is only the third reported HAL case with a normal serum level of AFP and AFP-negative immunostaining [2,3].

An equally intriguing aspect of this case was that the coinciding colorectal adenocarcinoma that resolved following chemoradiation. To our knowledge, this is the second case of HAL occurring simultaneously with a colorectal adenocarcinoma. The unremarkable AFP features of our case distinguishes it from the previous case. The other case of HAL with a coinciding colorectal adenocarcinoma had elevated serum levels of AFP, but these levels did not correlate with treatment response or patient outcome [4]. Given the findings of these two cases, AFP may not be a reliable tumor marker for HAL when a concurrent colorectal adenocarcinoma is present.

An unfortunately defining characteristic of HAL is the dismal prognosis of the disease. The majority of HAL cases share the poor prognostic factors of large primary tumor, advanced stage at presentation, high risk of recurrence, vascular endothelial growth factor (VEGF) expression, vascular invasion, and frequent hepatic metastases [6,7]. Most literature suggests that stage at presentation is the most important prognostic factor for HAL [8,9]. Additionally, three cases reported by Hiroshima et al. showed that AFP-positive immunostaining lung carcinomas had significantly more proliferative activity than those that did not stain for AFP, which suggests AFP expression might also be an important prognostic factor [10].

Given the poor prognosis and aggressive nature of HAL, molecular testing should be utilized by clinicians to explore targeted treatment options for HAL cases, especially those involving synchronous or refractory tumors. Notably, one previous case of HAL had the ALK gene rearrangement and responded briefly to crizotinib [3]. Our patient's tumor was negative for the ALK translocation and EGFR mutations, so no targeted therapies were used. 
Lastly, it is important to note the histological characteristics that discriminate HAL from metastatic hepatocellular carcinoma (HCC). A 14-marker immunohistolchemical panel was created by Haninger et al. in order to aid in this process [2]. In the 5 cases examined by Haninger et al., only HAL was found to stain for CK5/6 (1/5), CK7 (3/5), CK19 (4/5), CK20 (1/5), HEA125 (5/5), MOC31 (5/5), monoclonal CEA (3/5) and napsin A (1/5) [2]. Our patient's tumor only stained positive for CK7 in addition to characteristic markers of hepatocellular differentiation (HepPar1, HSP-70, and glypican-3). Therefore, this panel did not significantly aid us in distinguishing HAL from HCC.

In sum, we believe our diagnosis of HAL is supported by the immunohistochemical evidence of hepatic differentiation along with the morphologic features that mimic HCC, the lack of clinical or radiologic evidence of a liver tumor, and the fact that our patient had no history of HBV, HCV, or cirrhosis. We hope this report raises awareness of HAL and aids clinicians in distinguishing it from metastatic HCC. Given the poor prognosis of HAL, molecular testing should be utilized by clinicians to explore targeted treatment options. Specifically, the ALK rearrangement and crizotinib therapy has been documented in an AFP-negative HAL. Lastly, clinicians should be familiar with the histological characteristics that discriminate HAL from HCC and know that the diagnosis of HAL does not require elevated AFP serum levels or immunohistochemical evidence of AFP.

\section{Acknowledgments}

We thank all of our colleagues who helped us in the care of the patient. Additionally, the authors report no relevant conflict of interests and have no relevant funding sources to report.

\section{References}

1. Metzgeroth G, Ströbel P, Baumbusch T, Reiter A, Hastka J (2010) Hepatoid adenocarcinoma - review of the literature illustrated by a rare case originating in the peritoneal cavity. Onkologie 33: 263-269.[Crossref]

2. Haninger DM, Kloecker GH, Bousamra Ii M, Nowacki MR, Slone SP (2014) Hepatoid adenocarcinoma of the lung: report of five cases and review of the literature. Mod Pathol 27: 535-542.[Crossref]

3. Khozin S, Roth MJ, Rajan A, Smith K, Thomas A, et al. (2012) Hepatoid carcinoma of the lung with anaplastic lymphoma kinase gene rearrangement. J Thorac Oncol 7: e29-31.[Crossref]

4. Valentino F, Torchio M, Morbini P, Danoya M (2012) Synchronous presentation of hepatoid alpha-fetoprotein-producing lung cancer and colorectal adenocarcinoma. Tumori 98: 130e-134e. [Crossref]

5. Che YQ, Wang S, Luo Y, Wang JB, Wang LH (2014) Hepatoid adenocarcinoma of the lung: Presenting mediastinal metastasis without transfer to the liver. Oncol Lett 8: 105-110.[Crossref]

6. Ishikura H, Kanda M, Ito M, Nosaka K, Mizuno K (1990) Hepatoid adenocarcinoma: a distinctive histological subtype of alpha-fetoprotein-producing lung carcinoma. Virchows Arch A Pathol Anat Histopathol 417: 73-80.[Crossref]

7. Ishikura H, Kishimoto T, Andachi H, Kakuta Y, Yoshiki T (1997) Gastrointestinal hepatoid adenocarcinoma: venous permeation and mimicry of hepatocellular carcinoma. A report of 4 cases. Histopathology 31: 47-54. [Crossref]

8. Hayashi Y, Takanashi Y, Ohsawa H, Ishii H, Nakatani Y (2002) Hepatoid adenocarcinoma in the lung. Lung Cancer 38: 211-214.[Crossref]

9. Carlinfante G, Pia Foschini M, Pasquinelli G, Scott R, Cavazza A (2000)Hepatoid carcinoma of the lung: a case report with immunohistochemical, ultrastructural and in situ hybridization findings. Histopathology 37: 85-94. [Crossref]

10. Hiroshima K, Iyoda A, Toyozaki T, Haga Y, Baba M, et al. (2002) Alpha-fetoproteinproducing lung carcinoma: report of three cases. Pathol Int 52: 46-53.[Crossref]

Copyright: (C2016 Gutschenritter T. This is an open-access article distributed under the terms of the Creative Commons Attribution License, which permits unrestricted use, distribution, and reproduction in any medium, provided the original author and source are credited. 